\title{
Taxonomic Studies on Australian Psammoecus Latreille (Coleoptera, Silvanidae, Brontinae)
}

\author{
Michael KARNER \\ Voluntary Scientist, Sektion Entomologie I, Forschungsinstitut Senckenberg, \\ Senckenberganlage 25, D-60325 Frankfurt am Main, Germany. \\ Email: michael.karner@senckenberg.de \\ urn:lsid:zoobank.org:author:48E64F07-CDD3-4B4B-9C9A-24CBFCBBCB3D
}

\begin{abstract}
Two new species of Psammoecus Latreille, 1829 from Australia are described: Psammoecus australis sp. nov. and $P$. venustus sp. nov. A taxonomic revision and diagnoses for other Australian species are provided. Psammoecus obesus Grouvelle, 1919 is recorded from Australia for the first time. Two new synonyms are discovered: Psammoecus t-notatus Blackburn, $1908=$ P. amoenus Grouvelle, 1912 syn. nov.; Psammoecus vittifer Blackburn, $1903=$ P. concolor Grouvelle, 1919 syn. nov. A lectotype is designated for Psammoecus concolor Grouvelle, 1919.
\end{abstract}

Keywords. Coleoptera, Silvanidae, Psammoecus, Australia, taxonomy.

Karner M. 2020. Taxonomic Studies on Australian Psammoecus Latreille (Coleoptera, Silvanidae, Brontinae). European Journal of Taxonomy 723: 135-158. https://doi.org/10.5852/ejt.2020.723.1149

\section{Introduction}

Psammoecus Latreille, 1829 is a diverse genus within the Telephanini (Coleoptera: Silvanidae: Brontinae), with its highest diversity in the Oriental, Indo-Malayan, and Papua-Melanesian regions (Hetschko 1930). The genus remains poorly known, despite several studies in recent years (Pal 1985; Karner 2012, 2014; Yoshida \& Hirowatari 2014; Yoshida et al. 2018). Hetschko (1930) reported four species of Psammoecus from Australia: Psammoecus cruciger (Waterhouse, 1876) (as P. upsilon Blackburn, 1903), P. incertior Blackburn, 1903, P. t-notatus Blackburn, 1908 and P. vittifer Blackburn, 1903. By erroneously treating Psammoecus cruciger as a junior synonym of P. trimaculatus Motschulsky, 1858, Pal (1985) also listed P. trimaculatus as occuring in Australia.

A monographic revision of the entire genus would be highly desirable, but the sheer amount of work that such an endeavour would require renders it currently impossible for the present author. Instead, a step-wise approach is followed by dividing revisionary work into geographical areas of varying sizes, i.e., into manageable parts.

In the present paper, two new species of Psammoecus are described and two new synonymies are proposed; diagnoses and distribution data for other Australian species are provided. 


\section{Material and methods}

Observations and measurements were made using an Olympus SZX16 stereo microscope. Habitus photographs were taken with Canon EOS 650D and EOS 7D Mark II digital cameras and a Canon MP-E $65 \mathrm{~mm}$ macro objective. Higher magnifications were obtained with Mitutoyo M Plan Apo objectives $(10 \times$ and $20 \times$ ), with Asahi Takumar $200 \mathrm{~mm}$ and Carl Zeiss MC Sonnar $135 \mathrm{~mm}$ telephoto lenses as converging lenses. Photographs of genitalia were taken with Canon EOS 650D and EOS 7D Mark II digital cameras attached to an Olympus $\mathrm{CH}$ microscope. Images and image layers were processed with Zerene Stacker (Version 1.04), Adobe Lightroom 5.7 and GIMP (ver. 2.8.0) software.

A total of 383 specimens were studied.

Measurements were taken as follows: length, from apical margin of clypeus to apex of elytra; head width, across eyes; head length, from apical margin of clypeus to an imaginary line between hind margins of eyes; eye length, from anterior to posterior margin; antennal length, from base of $1^{\text {st }}$ antennomere to apex of $11^{\text {th }}$ antennomere; antennomere proportions were derived from actual measurements; pronotal width, across maximum width, excluding spines; pronotal length, from anterior to posterior margin; widths of anterior and posterior pronotal margins, across anterior and posterior denticles, respectively; elytral width, across maximum joint width; elytral length, along suture including scutellar shield. Temple angles were measured as described in Karner (2014).

In addition to the definitions proposed by Karner (2014), some further remarks on diagnostic characters are necessary:

The shape of the punctures on the vertex and pronotum has considerable diagnostic value. Punctures can be round or narrowed; sometimes they are represented by slit-like, very deep impressions. If punctures are narrowed, the term 'elongate' indicates an elongation in the direction of the body axis (e.g., Fig. 2B, punctation on vertex), while 'widened' indicates an elongation perpendicular to the body axis (e.g., Fig. $2 \mathrm{C}$, punctation on pronotum).

Assessing the width of elytral striae (i.e., the width of strial punctures) can be difficult due to the presence of darkened areas surrounding the individual punctures. The width of these areas does not indicate the actual width of the striae. The strial puncturation should therefore be assessed under oblique, diffused illumination. Strial width in the sense of this work is the width of rows of punctures as indicated by the impressed area of the elytral surface.

Male genitalia vary greatly in shape and in the degree of sclerotization. It was found that strongly sclerotised types often have a more complex three-dimensional structure, whereas weakly sclerotised types are in general dorso-ventrally flattened. Lateral views of male genitalia are only provided if important diagnostic characters were found. Accordingly, both ventral and dorsal views are only depicted if the ventral aspect alone does not provide a sufficiently clear view of the shape of the median lobe.

For holotypes and type material of previous authors, label data are given verbatim, including atypical use of punctuation and spaces. The labels are cited beginning with the uppermost one, the respective lines of text are separated by '|'. Comments on label colours, label shapes, etc., regarding the respective specimen are included in square brackets. Data are condensed for paratypes of newly described species and for other material.

Genitalia were prepared as described by Karner (2012). For photography, isolated aedeagi were placed in Kaiser's glycerol gelatin (Carl Roth $\mathrm{GmbH}$ ) to enable precise orientation. After study, dissected parts were embedded in dimethylhydantoin formaldehyde resin on cellulose acetate labels and attached to the pins of the respective specimens. 


\section{Institutional abbreviations}

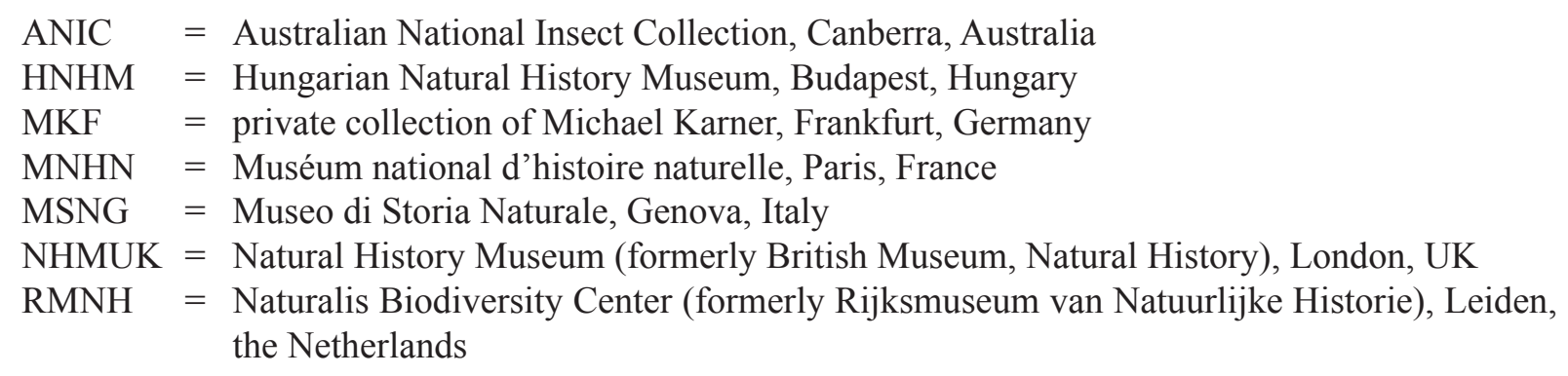

\section{Results}

Class Insecta Linnaeus, 1758

Order Coleoptera Linnaeus, 1758

Superfamily Cucujoidea Latreille, 1802

Family Silvanidae Kirby, 1837

Subfamily Brontinae Erichson, 1845

Tribe Telephanini LeConte, 1861

Genus Psammoecus Latreille, 1829

Psammoecus cruciger (Waterhouse, 1876)

Fig. 1

Telephanus cruciger Waterhouse, 1876: 125.

Psammoecus upsilon Blackburn, 1903: 155.

Psammoecus cephalotes Grouvelle, 1919: 20.

Psammoecus cruciger - Grouvelle 1908: 476. — Arrow 1927: 44. — Hetschko 1930: 82.

Psammoecus cephalotes - Arrow 1927: 44. - Hetschko 1930: 82. — Pal 1985: 41.

Psammoecus upsilon - Arrow 1927: 44.

Psammoecus ypsilon - Hetschko 1930: 82. — Pal 1985: 41 (misspellings).

non Psammoecus trimaculatus - Grouvelle 1908: 476 (misidentification). — Pal 1985: 41.

\section{Diagnosis}

The following combination of character states distinguishes this species: body oval (Fig. 1A) length 1.97$2.73 \mathrm{~mm}$; eyes (Fig. 1B) large, evenly rounded; temples distinct, narrowed evenly immediately behind eyes; frontal grooves flat, slightly curved, short, reaching anterior fifth of eyes; antennae stout (Fig. 1C), antennomere 6 sometimes slightly darkened, antennomeres 7-10 distinctly darkened, antennomere 11 whitish yellow; punctation on head and pronotal disc dense, microsculpture absent, punctures round, somewhat smaller than an eye facet diameter, pubescence on head and pronotum moderate; pronotum (Fig. 1B) widest at anterior third, pronotal punctation moderate, punctures widened, about as wide as eye facet diameter, lateral pronotal margins with four, sometimes only three teeth of moderate size, anterior and posterior denticles present, small; elytra (Fig. 1A) widest at middle, elytra with piceous maculae: wide band near middle, posterior half of suture and elytral apex darkened, humeral area often slightly to distinctly darkened; elytral pubescence short, longer setae along anterior half of interstice between $9^{\text {th }}$ and $11^{\text {th }}$ stria; male genitalia (Fig. 1D-F) moderately sclerotised, with short, blunt median lobe that bears distinct, complex sclerotizations in apical part of internal sac, parameres short, bearing three distinct, long setae, parameres fused with tegmen, inner margins fused medially at flat angle, almost forming arc. 


\section{Material examined}

Types

PAPUA NEW GUINEA • 9 , holotype of Telephanus cruciger Waterhouse, 1876; Dory; "Type" [round label with red border]; "59 58. Dory. | New Guinea"; "Telephanus | cruciger, | (Type) C.Waterh.”. [5958 refers to 703 Coleoptera specimens collected by Alfred Russel Wallace in Dory, New Guinea, and puchased by NHMUK from Stevens' Auction House in 1859 (M.V.L. Barclay pers. comm.)]; NHMUK.

AUSTRALIA - 9 , holotype of Psammoecus upsilon Blackburn, 1903 (= P. ypsilon sensu Hetschko 1930); [Northern Queensland?]; “T. | 7223 | N. Qv [?]" [written on mounting label, last line in red ink]; "Type | H. T." [round label with red border]; "Psammoecus | upsilon, Blackb."; "Australia. | Blackburn Coll. | B.M. 1910-236.”; NHMUK.

AUSTRALIA - 9 , holotype of Psammoecus cephalotes Grouvelle, 1919; Port Darwin; "Type" [round label with red border]; "Port Darwin. | 92-2."; "4716"; "Psammoecus | cephalotes | G. Grouv" [Grouvelle's hand]; NHMUK.

\section{Other material}

AUSTRALIA - Queensland - Cairns • 3 specs; Aug. 1947; ANIC $\bullet 4$ specs; ANIC $\bullet 6$ specs; Sep.

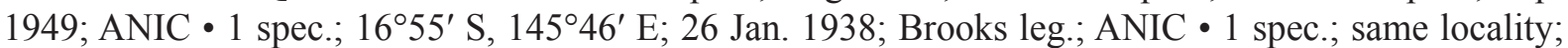
18 Apr.-14 May 1965; Brooks leg.; at indoor light; ANIC • 1 spec.; 25 Mar. 1965; Balogh leg.; HNHM • 1 spec.; Little Mulgrave National Park; 16 Dec. 1967; Brooks leg.; ANIC • 1 spec.; Russell River Xing; $17^{\circ} 26^{\prime} \mathrm{S}, 145^{\circ} 54^{\prime} \mathrm{E}$; 11 Jun. 1992; Reid leg.; weedy rainforest, beating Hibiscus; ANIC • 10 specs; Edge Hill; 16 $55^{\prime}$ S, $145^{\circ} 46^{\prime}$ E; 9 Aug. 1968; Balogh and Loksa leg.; HNHM. - Cassowary Coast Region - 1 spec.; Cardstone; 1 Jan. 1966; Hyde leg.; ANIC • 1 spec.; same locality; 2 Feb. 1966; Brooks leg.; ANIC • 6 specs; same locality; 10-13 Mar. 1966; Hyde leg.; ANIC • 1 spec.; same locality; 14 Nov. 1966; Brooks leg.; ANIC • 4 specs; same locality; 15 Nov. 1966; Brooks leg.; ANIC • 1 spec.; same locality; 16 Nov. 1966; Brooks leg.; ANIC • 2 specs; same data as for preceding; MKF 1 spec.; same locality; 19 Nov. 1966; Brooks leg.; ANIC • 1 spec.; same locality; 28 Nov. 1966; Brooks leg.; ANIC - 1 spec.; same locality; 1-2 Dec. 1966; Brooks leg.; ANIC • 1 spec.; same locality; 5-6 Dec. 1966; Brooks leg.; ANIC • 1 spec.; same locality; 20 Jan. 1967; Hyde leg.; ANIC • 4 specs; Hinchinbrook Island, Gayundah Creek; 8-9 Nov. 1984; Marks leg.; light trap; ANIC • 1 spec.; same locality; 15-17 Nov. 1984; Marks leg.; light trap; ANIC• 1 spec.; Innisfail, Seymour Range; 6 Nov. 1966; Britton leg.; rainforest at light; ANIC. - Mackay Region - 4 specs; Finch Hatton, Cattle Ck Xing; 9 Jun. 1992; Reid leg.; beaten from flowering Eucalyptus; ANIC • 1 spec.; Bucasia; 3 Jan. 2007; Sandery leg.; ANIC. -

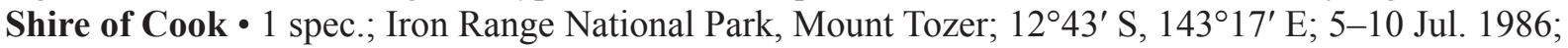
Weir and Calder leg.; at light; ANIC. - Shire of Douglas • 1 spec.; Daintree; 29 Oct. 1966; Britton leg.; dead leaves edge of rainforest; ANIC 1 spec.; same data as for preceding; MKF. - Shire of Mareeba •

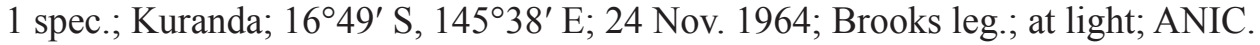

MALAYSIA • 2 specs; Sarawak, W Kuching, Mt Matang; Dec. 1913; Bryant leg.; NHMUK • 1 spec.; same data as for preceding; MKF.

PAPUA NEW GUINEA • 1 spec.; NE Madang, Gogol River; 17 Sep. 1969; Balogh leg.; HNHM • 1 spec.; Western Province, SE Kiunga; 23 Jul.-2 Aug. 1969; Balogh leg.; HNHM.

\section{Distribution}

Psammoecus cruciger has been found in Australia (Queensland), Malaysia and Papua New Guinea. 

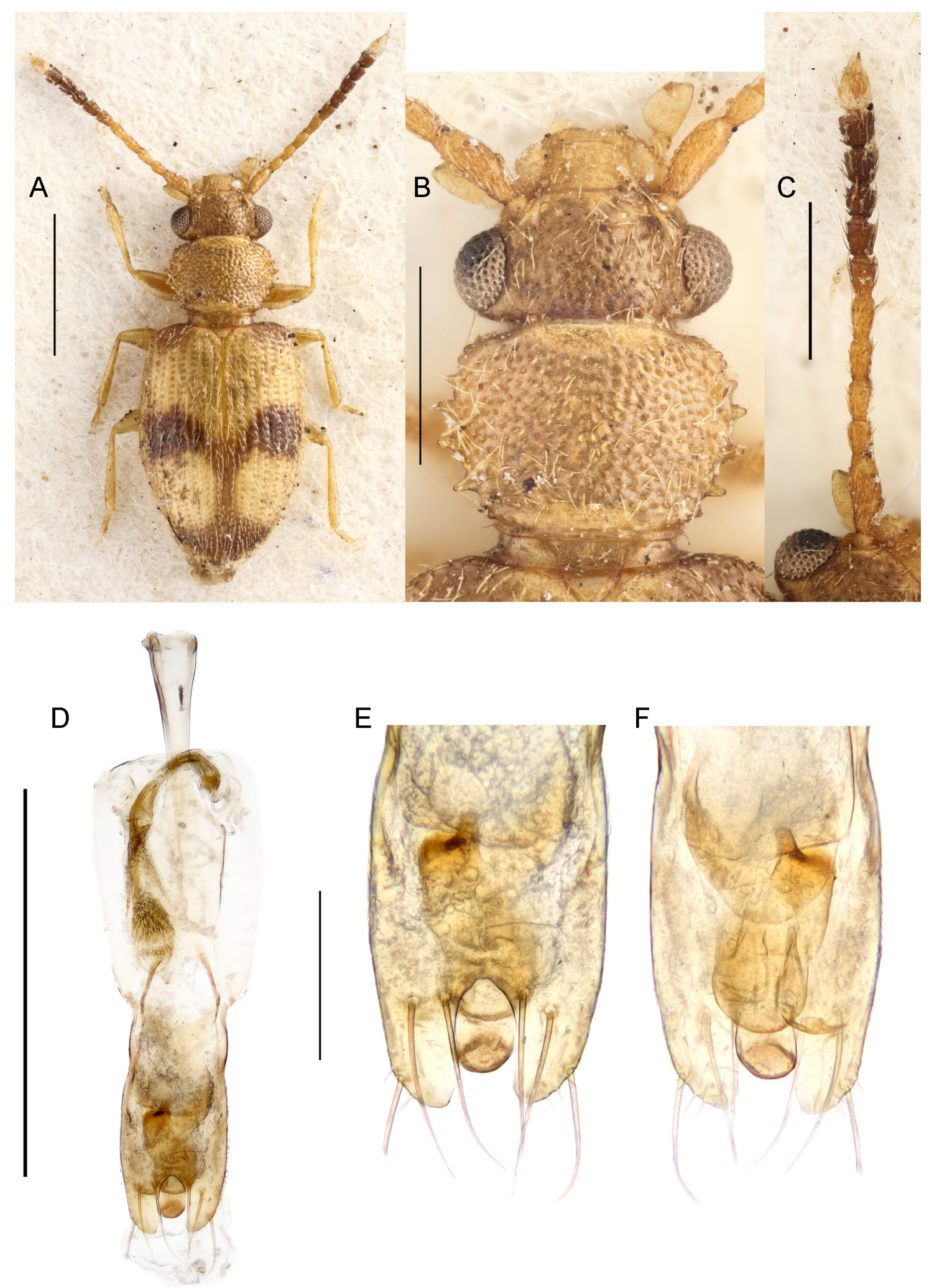

Fig. 1. Psammoecus cruciger (Waterhouse, 1876). A-C. Holotype, $q$ (NHMUK). A. Habitus. B. Head and pronotum. C. Left antenna. D-F. Specimen from Queensland, $\widehat{O}$ (ANIC). D. Aedeagus, ventral view. E. Parameres, ventral view. F. Median lobe, dorsal view. Scale bars: A $=1.0 \mathrm{~mm}$; B-D $=0.5 \mathrm{~mm}$; $\mathrm{E}-\mathrm{F}=0.1 \mathrm{~mm}$. 


\section{Remarks}

Grouvelle (1908) erroneously synonymised P. cruciger with P. trimaculatus Motschulsky, 1858. In his revision of Indian Psammoecus, Pal (1985) agreed with this, treating P. upsilon ("P. ypsilon") and $P$. cephalotes as "probable synonyms" of $P$. trimaculatus. Arrow (1927) did not adopt Grouvelle's synonymization of $P$. cruciger with $P$. trimaculatus. Instead, he regarded $P$. upsilon and $P$. cephalotes as junior synonyms of $P$. cruciger. Examination of type material confirmed the synonymy according to Arrow. There are few similarities to $P$. trimaculatus; the structure of the male genitalia indicates a very close relationship to $P$. simonis Grouvelle, 1882. Both $P$. trimaculatus and $P$. simonis were redescribed by Yoshida \& Hirowatari (2014).

\section{Psammoecus incertior Blackburn, 1903}

Fig. 2

Psammoecus incertior Blackburn, 1903: 154.

Psammoecus incertior - Hetschko 1930: 83.

\section{Diagnosis}

The following combination of character states distinguishes this species: body elongate-oval (Fig. 2A), length 2.86-3.30 mm; eyes large (Fig. 2B), unevenly rounded with distinctly stronger curvature posteriorly; temples very short; frontal of moderate length, diverging anteriorly, almost parallel posteriorly, reaching anterior $2 / 5$ of eyes; antennae somewhat short (Fig. 2D), stout, antennomere 8 castaneous, antennomeres 9-10 piceous; antennomere 11 yellowish brown; punctation on vertex moderate (Fig. 2B), punctures strongly elongate, almost twice as long as eye facet diameter, microsculpture absent, pubescence moderate; pronotum widest at anterior third (Fig. 2C), lateral pronotal margins with six small but distinct teeth; teeth II, V, and VI somewhat larger than others, anterior denticles small, posterior denticles very small; pronotal punctation moderate, punctures distinctly widened, microsculpture absent, pubescence moderate, setae directed medially; elytra widest just in front of middle (Fig. 2A), with large brown medio-lateral maculae and small darkened area at posterior third of suture, striae as wide as interstices, pubescence moderate, microsculpture absent; male genitalia strongly sclerotised (Fig. 2E-H), median lobe slender, evenly narrowed towards apex, tip blunt in dorsal view; curved ventrally with sharp tip in lateral view; parameres short, wide, with short protrusion at postero-lateral margin, two long setae near protrusion and few short setae along inner margins.

\section{Material examined}

\section{Holotype}

AUSTRALIA • O'; "Type | H.T.” [round label with red border]; "Australia. | Blackburn Coll. | B.M.1910236."; "Psammoecus | incertior Blackb."; NHMUK.

\section{Other material}

AUSTRALIA-Queensland • 1 spec.; Cairns; $16^{\circ} 55^{\prime}$ S, $145^{\circ} 46^{\prime}$ E; May 1947; ANIC • 2 specs; Cassowary

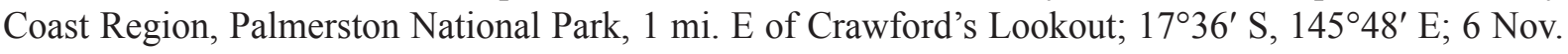
1966; Britton leg.; under bark; ANIC • 2 specs; Shire of Mareeba, Kuranda; $16^{\circ} 49^{\prime}$ S, $145^{\circ} 38^{\prime}$ E; Oct.

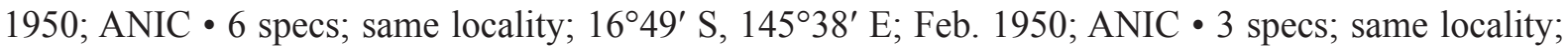
Feb. 1909; Bryant leg.; NHMUK • 1 spec.; same data as for preceding; MKF 1 spec.; Shire of Mareeba, 6 km NE of Kuranda, Black Mt Road; 16² 46' S, 14540' E; 21 May 1976; Britton leg.; MKF • 2 specs; Tablelands, 6.8 km W of Atherton, Russel River Crossing; 17²6’ S, 145 54' E; 11 Jun. 1992; Reid leg.; rainforest, beaten; ANIC 11 spec.; Townsville Regional City, Magnetic Island; $19^{\circ} 8^{\prime}$ S, $146^{\circ} 50^{\prime}$ E; Jul. 1921; Carter leg.; ANIC. 

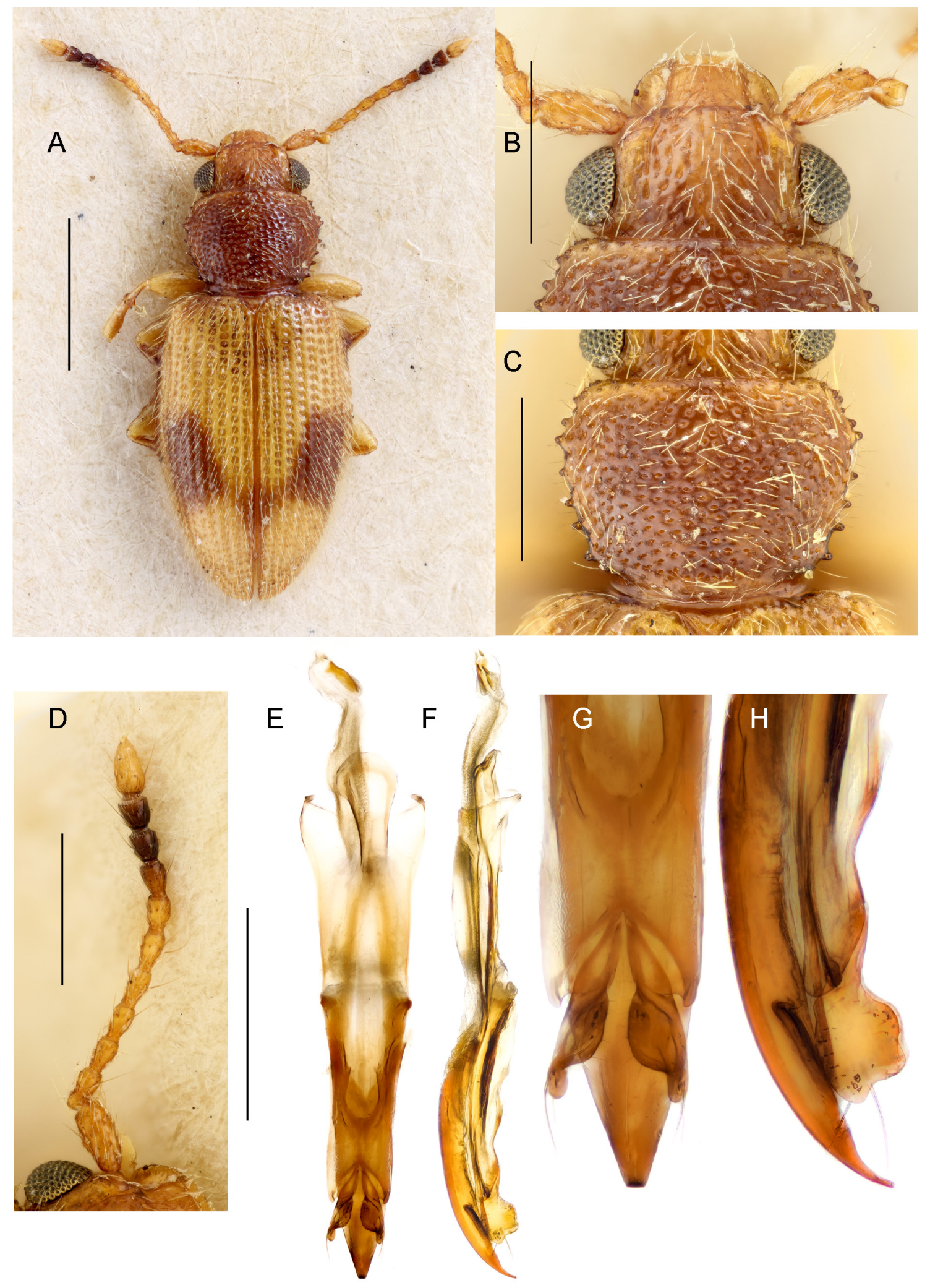

Fig. 2. Psammoecus incertior Blackburn, 1903, $\hat{\jmath}$. A-C. Holotype (NHMUK). A. Habitus. B. Head. C. Pronotum. D-H. Specimen from Queensland (ANIC). D. Left antenna. E. Aedeagus, ventral view. F. Aedeagus, lateral view. G. Parameres, ventral view. H. Median lobe and parameres, lateral view. Scale bars: A $=1.0 \mathrm{~mm}$; B-F $=0.5 \mathrm{~mm}$; $\mathrm{G}-\mathrm{H}=0.2 \mathrm{~mm}$. 


\section{Distribution}

Australia (Queensland).

\section{Remarks}

The colouration of this species is rather variable, but the general pattern remains constant. A very distinct character is the peculiar shape of the male parameres, which differs considerably from all other known species of Psammoecus.

\section{Psammoecus obesus Grouvelle, 1919}

Fig. 3

Psammoecus obesus Grouvelle, 1919: 11.

Psammoecus obesus - Hetschko 1930: 83.

\section{Diagnosis}

The following combination of character states distinguishes this species: body elongate-oval (Fig. 3A), length 3.08-3.29 mm; eyes large (Fig. 3B), irregularly rounded with stronger curvature posteriorly; temples distinct, short, strongly curved; frontal grooves distinct, diverging anteriorly, parallel near end; reaching anterior $2 / 5$ of eyes; antennae stout (Fig. 3D), antennomere 8 slightly darkened, antennomeres 9-10 darkened, antennomere 11 bright yellowish-brown; punctation on vertex moderate, punctures distinctly elongate, as large as eye facet diameter; microsculpture on vertex absent, pubescence moderate; pronotum widest just in front of middle (Fig. 3C), lateral pronotal margins with five teeth; tooth I slightly and tooth V distinctly bigger than II-IV, distance between I and II wider than distances between other teeth, anterior and posterior denticles present, small; pronotal punctation dense, punctures as large as on vertex, distinctly widened, microsculpture absent, pubescence moderate, setae directed medially; elytra widest at middle (Fig. 3A), with transverse brown maculae behind middle, interstices distinctly wider than striae, pubescence moderate, microsculpture absent; male genitalia (Fig. 3E-H) strongly sclerotised, median lobe in dorsal view wide, narrowed abruptly near ostium, apical end well rounded, in lateral view also narrowed abruptly, with sharp apical end; parameres in ventral view very wide, inner margins, apex and apical third of lateral margins with distinct setae; in lateral view with very wide basis, narrowed abruptly towards apex with dorsal margin almost forming angle.

\section{Material examined}

\section{Holotype}

INDONESIA • ; Maluku, Seram; Pascoe leg.; "HOLO - | Type" [round label with red border]; "Ceram" [blue, elliptic label]; "Pascoe | Coll. | 93-60"; "Psammoecus | obesus | ty. Grouv" [Grouvelle's hand]; "HOLOTYPUS | Psammoecus | obesus Grouv. 1919| det. R.G. Booth 2013”; NHMUK.

\section{Other material}

AUSTRALIA • 1 spec.; Queensland, Cairns; 1655' S, 14546' E; 1942; Perkins leg.; NHMUK • 1 spec.; Queensland, Cassowary Coast Region, 10 km NE of Tully; Nov. 1994; C. Reid leg.; rainforest; ANIC - 1 spec.; Queensland, Cassowary Coast Region, Cardstone; 1-2 Dec. 1966; J.G. Brooks leg.; ANIC • 1 spec.; Queensland, Shire of Cook, McIlwraith Range; 500 m a.s.1.; $13^{\circ} 45^{\prime}$ S, $143^{\circ} 22^{\prime}$ E; 27 Jun. -12 Jul. 1989; T.A. Weir leg.; at light; ANIC • 1 spm; Queensland, Shire of Douglas, Daintree; 29 Oct. 1966; E. Britton leg.; dead leaves, edge of rainforest; ANIC • $1 \mathrm{spm}$; same data as for preceding; MKF.

INDONESIA • 2 specs; West Papua, Waigeo, Camp Nok; $2500 \mathrm{ft}$ a.s.1.; $0^{\circ} 12^{\prime} \mathrm{S}, 130^{\circ} 50^{\prime}$ E; Apr. 1938; Cheesman leg.; NHMUK. 

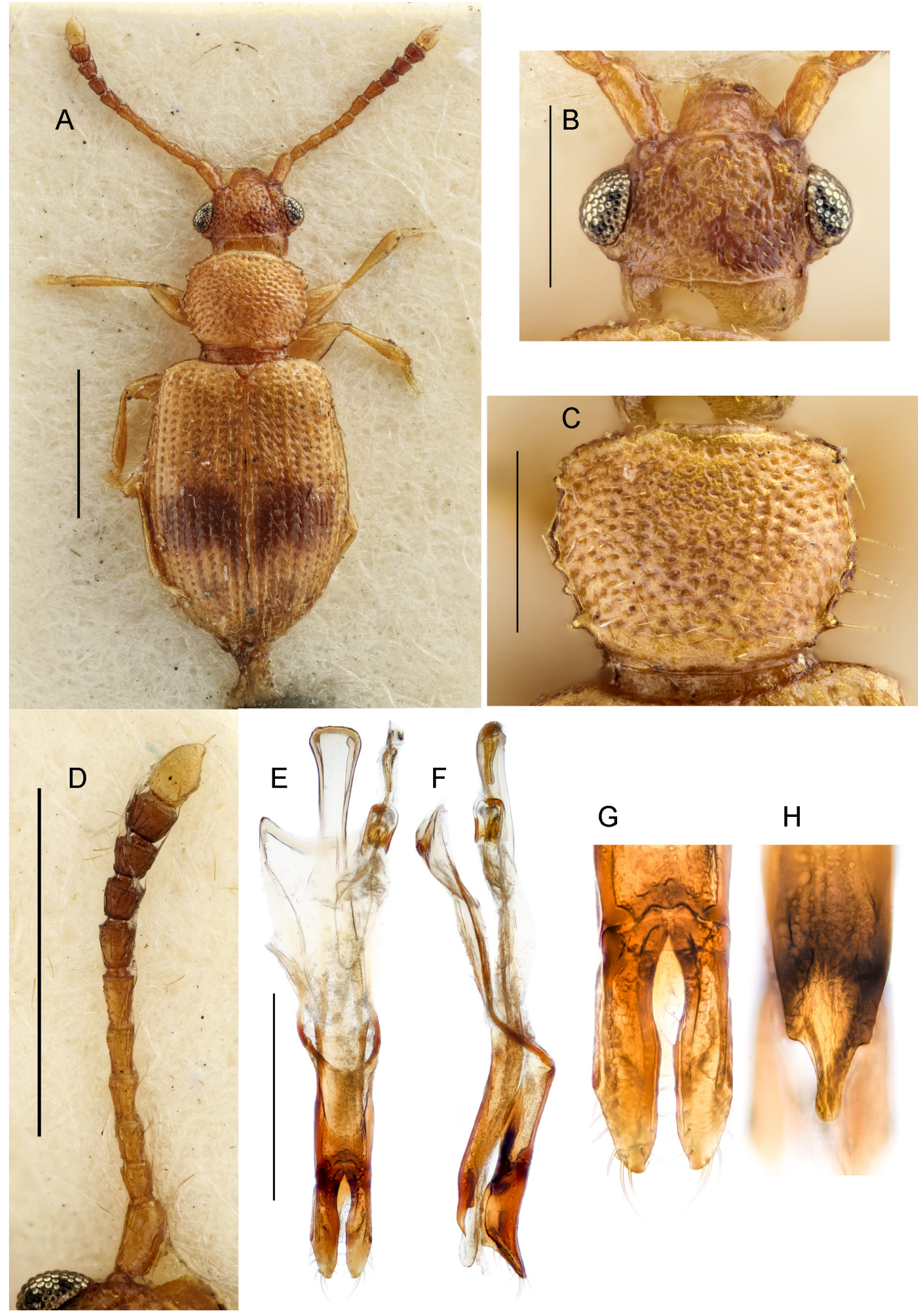

H

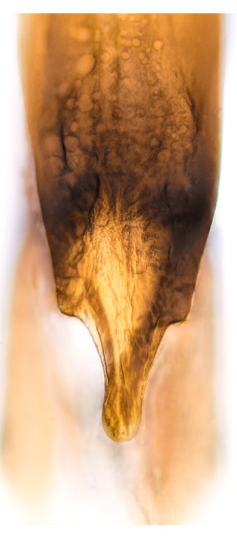

Fig. 3. Psammoecus obesus Grouvelle, 1919. A-D. Holotype, $q$ (NHMUK). A. Habitus. B. Head. C. Pronotum. D. Left antenna. E-H. Specimen from Queensland, ô (ANIC). E. Aedeagus, ventral view. F. Aedeagus, lateral view. G. Parameres, ventral view. H. Median lobe, dorsal view. Scale bars: A, D = $1.0 \mathrm{~mm} ; \mathrm{B}-\mathrm{C}, \mathrm{E}-\mathrm{F}=0.5 \mathrm{~mm} ; \mathrm{G}-\mathrm{H}=0.2 \mathrm{~mm}$. 


\section{Distribution}

The species has been found in Australia (Queensland) and Indonesia.

\section{Remarks}

Psammoecus obesus is closely related to P. hirsutus Grouvelle, 1883, P. decoratus Grouvelle, 1919, and P. signatus Grouvelle, 1919. It differs from all three by the shape of the male genitalia.

\section{Psammoecus t-notatus Blackburn, 1903}

Fig. 4

Psammoecus t-notatus Blackburn, 1903: 154.

Psammoecus amoenus Grouvelle, 1912: 92. Syn. nov.

Psammoecus t-notatus - Hetschko 1930: 84.

\section{Diagnosis}

The following combination of character states distinguishes this species: body oval (Fig. 4A), length $2.35-2.83 \mathrm{~mm}$; eyes of moderate size (Fig. 4B), slightly unevenly rounded with somewhat stronger curvature posteriorly; temples distinct, evenly rounded; frontal grooves short, slightly curved, reaching anterior third of eyes; antennae slender (Fig. 4D), antennomeres 9-10 darkened; antennomere 11 testaceous; punctation on vertex moderate (Fig. 4B), punctures round, slightly smaller than eye facet diameter; microsculpture on vertex absent, pubescence moderate; pronotum (Fig. 4C) often brown with testaceous discal area of variable size, sometimes entire pronotum testaceous; widest at anterior third, lateral pronotal margins with four distinct teeth; teeth I and II small, III and IV larger; anterior and posterior denticles present, distinct; pronotal punctation dense, punctures slightly widened anteromedially, microsculpture absent, pubescence moderate, setae directed antero-medially; elytra widest at middle (Fig. 4A), with transverse piceous maculae closely behind middle, posterior part of suture, elytral apex and, in most specimens, humeral area darkened, interstices slightly wider than striae, pubescence moderate, microsculpture absent; male genitalia (Fig. 4E-F) comparatively weakly sclerotised, median lobe slender, evenly narrowed towards apex, tip of apex blunt, tegmen with wide, very weakly sclerotised basis, paramere long, slender, club-shaped, inner margin of widened basis with numerous setae, along inner margins with few short, thin setae, apex with long seta.

\section{Material examined}

\section{Types}

AUSTRALIA • + , holotype of Psammoecus t-notatus Blackburn, 1903; "Type | H.T." [round label with red border]; "Australia. | Blackburn Coll. | B.M.1910-236."; "Psammoecus | T-notatus., Blackb."; NHMUK.

VIETNAM • ^’ holotype of Psammoecus amoenus Grouvelle, 1912; Annam, Phúc Son; “Annam | PhucSon | Nov. Dez. | H. Fruhstorfer"; "Psammoecus | amoenus | G. Grouv" [Grouvelle's hand]; "N.L.M. 34. | p. 92."; "Museum Leiden | Psammoecus | amoenus | Det: Grouv."; "Type" [red label]; RMNH.

\section{Other material}

AUSTRALIA • 2 specs; Queensland, Cairns; $16^{\circ} 55^{\prime}$ S, $145^{\circ} 46^{\prime}$ E; ANIC $・ 3$ specs; Queensland, Cairns, Bellenden Ker Range, 1 km south of Cable Tower; 1054 m a.s.1.; 17 Oct.-5 Nov. 1981; QLD Museum leg.; rainforest, malaise trap; ANIC • 1 spec.; Queensland, Cassowary Coast Region, Cardstone; 14 Nov. 1966; Brooks leg.; ANIC • 1 spm; same locality; 15 Nov. 1966; Brooks leg.; ANIC • 1 spm; same data as for preceding; MKF • 1 spec.; same locality; 20 Nov. 1966; Hyde leg.; MKF • 2 specs; same 


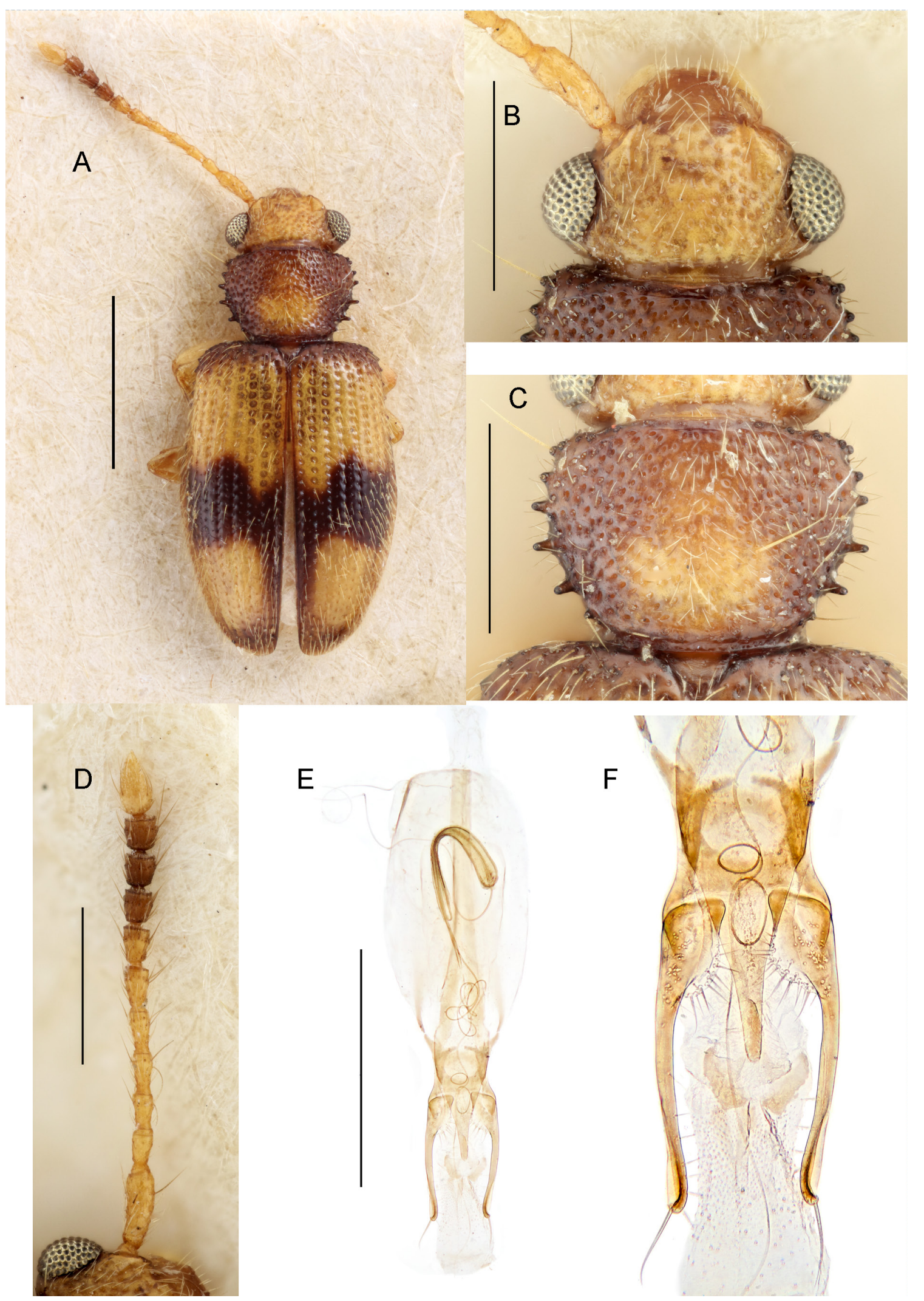

Fig. 4. Psammoecus t-notatus Blackburn, 1903. A-D. Holotype, $q$ (NHMUK). A. Habitus. B. Head. C. Pronotum. D. Left antenna. E-F. Specimen from Papua New Guinea, $\widehat{\partial}$ (NHMUK), aedeagus, ventral view. Scale bars: $A=1.0 \mathrm{~mm}$; $-\mathrm{E}=0.5 \mathrm{~mm} ; \mathrm{F}=0.2 \mathrm{~mm}$. 
locality; 1 Feb. 1966; Hyde leg.; ANIC• 1 spec.; same locality; 2 Feb. 1966; Hyde leg.; ANIC • 1 spec.; Queensland, Shire of Douglas, Daintree, 4 mi. S. of Daintree; 29 Oct. 1966; Britton leg.; edge of rainforest on dead leaves; ANIC -2 specs; Queensland, Shire of Mareeba, Kuranda; $16^{\circ} 49^{\prime}$ S, 145 $38^{\prime}$ E; Dec. 1964; Brooks leg.; ANIC • 4 specs; same locality; Mar. 1950; Brooks leg.; ANIC• 1 spec.; Queensland, Shire of Mareeba, Kuranda, Black Mountain Road 10 mi. N of Kuranda; 16 ${ }^{\circ} 46^{\prime}$ S, 145 $45^{\prime}$ E; 8 Mar. 1969; Brooks leg.; ANIC • 1 spec.; Queensland, Tablelands Region, Ravenshoe; 25 Dec. 1937; ANIC.

FIJI -2 specs; Taveuni; 10 Dec. 1923; Evans leg.; NHMUK $・ 1 \mathrm{spm}$; same data as for preceding; MKF • 2 specs; Rotuma Island, Noatau; 12²9.9' S, 177².82' E; 26 Apr. 1971; Robinson leg.; at light; NHMUK.

INDONESIA • 6 specs; Java; NHMUK • 2 specs; Java; Wallace leg.; NHMUK • 1 spec.; Sumatra; Doherty leg.; NHMUK.

MALAYSIA • 8 specs; Pahang, Taman Negara; 1-13 Mar. 1984; Jessop leg.; at light; NHMUK $\bullet 2$ specs; same data as for preceding; MKF • 5 specs; Sarawak, Mt Matang; Dec. 1913; Bryant leg.; NHMUK • $1 \mathrm{spm}$; same data as for preceding; MKF 11 spec.; Sarawak, Muara Tuang, Kampung Quop; Feb.-Mar. 1914; Bryant leg.; NHMUK.

PAPUA NEW GUINEA • 8 specs; Madang, R. Buru, $90 \mathrm{~m}$ NW of Lae; $1200 \mathrm{ft}$ a.s.1.; 513' S, $145^{\circ} 48^{\prime}$ E; 1 Oct. 1964; Bacchus leg.; at light; NHMUK • 1 spec.; Morobe, Lae; 644' S, $147^{\circ} 0^{\prime}$ E; 10 Dec. 1964; Bacchus leg.; NHMUK • 11 specs; Morobe, Finisterre Range, Budemu; $4000 \mathrm{ft}$ a.s.1.; $5^{\circ} 48^{\prime} \mathrm{S}, 146^{\circ} 6^{\prime} \mathrm{E}$; 15-24 Oct. 1964; Bacchus leg.; at light; NHMUK • 1 spm; same data as for preceding; MKF 12 specs; Morobe, Finisterre Range, Damanti; $3550 \mathrm{ft}$ a.s.1.; 5²48' S, 146 $6^{\prime}$ E; 2-11 Oct. 1964; Bacchus leg.; at light; NHMUK • $1 \mathrm{spm}$; same data as for preceding; MKF • 1 spec.; Morobe, Herzog Mountains, Vagau; $4000 \mathrm{ft}$ a.s.1.; 4-17 Jan. 1965; Bacchus leg.; NHMUK.

THAILAND • 1 spec.; Ranong; Doherty leg.; NHMUK.

VANUATU • 1 spec.; Malakula Island; $16^{\circ} 15^{\prime} \mathrm{S}, 167^{\circ} 30^{\prime}$ E; Mar.-Apr. 1929; Cheesman leg.; NHMUK - 2 specs; Espiritu Santo; $15^{\circ} 15^{\prime}$ S, 166 50' E; Aug. 1929; Cheesman leg.; NHMUK • 2 specs; same locality; Aug.-Sep. 1929; Cheesman leg.; NHMUK・1 spm; same data as for preceding; MKF.

\section{Distribution}

Specimens of $P$. t-notatus have been recorded from Australia (Queensland), Fiji, Indonesia, Malaysia, Papua New Guinea, Thailand and Vanuatu.

\section{Remarks}

Psammoecus t-notatus is closely related to P. trimaculatus Motschulsky, 1858 and P. triguttatus Reitter, 1874. Most specimens can easily be recognised by their distinct colouration/colour pattern. The determination of unusually coloured specimens requires examination of male genitalia. Psammoecus t-notatus differs from P. trimaculatus by the distinctly longer apical part of the parameres; it differs from P. triguttatus by the elongate, slender median lobe and the large, wide basal part of the tegmen.

Psammoecus t-notatus has a remarkably large area of distribution. As for other species of the genus, records from Malaise traps and at light suggest high mobility and a tendency to accumulate near human settlements. This, combined with an association with plant detritus and/or fungi, is likely to facilitate distribution by human trade of agricultural goods. 
Psammoecus vittifer Blackburn, 1903

Fig. 5

Psammoecus vittifer Blackburn, 1903: 155.

Psammoecus concolor Grouvelle, 1919: 8. Syn. nov.

Psammoecus vittifer - Hetschko 1930: 84.

\section{Diagnosis}

The following combination of character states distinguishes this species: body elongate-oval (Fig. 5A), length $2.36-2.85 \mathrm{~mm}$; eyes of moderate size (Fig. 5B), unevenly rounded with distinctly stronger curvature posteriorly; temples very short; frontal grooves short, slightly curved, reaching anterior third of eyes; antennae short, stout (Fig. 5D), antennomere 6 slightly darkened, antennomeres 7-10 dark brown; antennomere 11 whitish; punctation on vertex dense (Fig. 5B), punctures strongly elongate, slightly longer than eye facet diameter, microsculpture absent, pubescence moderate; pronotum widest at anterior third (Fig. 5C), lateral pronotal margins with five small, but distinct teeth; I and V larger than II-IV, anterior denticles small, spread over larger distance and reaching anterior $1 / 6$ of lateral margins, posterior denticles very small; pronotal punctation dense, punctures distinctly widened, microsculpture absent, pubescence moderate, setae directed antero-medially; elytra widest at middle (Fig. 5A), with elongate brown maculae along anterior $2 / 3$ of margins and sutural area darkened near apex, striae wider than interstices, pubescence moderate, microsculpture absent; male genitalia strongly sclerotised (Fig. 5E-H), median lobe slender, evenly narrowed towards apex, tip blunt in dorsal and lateral view, parameres long, wide, apex thin, flattened and widened, lateral margin near apex with two long setae. Left paramere of holotype of $P$. vittifer (Fig. 5G) permanently bent ventrally, probably a drying artifact.

\section{Material examined}

\section{Types}

AUSTRALIA • 1 $\lesssim$, holotype of Psammoecus vittifer Blackburn, 1903; "Type | H.T." [round label with red border]; "Australia. | Blackburn Coll. | B.M.1910-236."; "Psammoechus [sic!] | vittiferus, Blackb.”; NHMUK.

INDONESIA • 1 o, lectotype (here designated) of Psammoecus concolor Grouvelle, 1919; Java; "Co- | type" [round label with yellow border]; "Java."; "Bowring. | 63.47*"; "Psammoecus | concolor Grouv." [not Grouvelle's hand]; Bowring leg.; MNHN • 1 क ; same data as for preceding; NHMUK.

Of P. concolor, Grouvelle (1919: 8) mentions two type specimens from Java in the collection of the NHMUK. Only one syntype ( $(+)$ could be located in the NHMUK collection; a further syntype $(\precsim)$ with identical data is stored in the MNHN collection, indicating that Grouvelle kept one specimen for his own collection. The MNHN specimen is hereby designated as lectotype.

\section{Other material}

AUSTRALIA - Queensland • 3 specs; Cairns Region, Cairns; Sep. 1949; ANIC • 3 specs; same locality; Nov. 1949; ANIC • 1 spec.; Cairns, Edge Hill; 1655' S, 145²6' E; 18 Apr.-14 May 1965; J.G. Brooks leg.; at indoor lights; ANIC • 4 specs; same locality; 11 Nov. 1967; J.G. Brooks leg.; ANIC • 1 spec.; same locality; 14 Oct. 1970; J.G. Brooks leg.; MKF • 1 spec.; Cassowary Coast Region, Cardstone; 25 Nov. 1966; J.G. Brooks leg.; ANIC • 1 spec.; same locality; 26 Nov. 1966; J.G. Brooks leg.; ANIC• 1 spec.; same locality; 29 Nov. 1966; J.G. Brooks leg.; ANIC • 1 spec.; same locality; 15-16 Jan. 1967; K. Hyde leg.; MKF 1 spec.; Cassowary Coast Region, W slopes of Seymour Range, near Innisfail, Dinner Creek Road; 6 Nov. 1966; E. Britton leg.; rainforest, at light; ANIC • 1 spec.; Cassowary Coast Region, Palmerston National Park, Tully-Cairns Powerline; 300 m a.s.1.; 6 Nov. 1966; E. Britton leg.; 

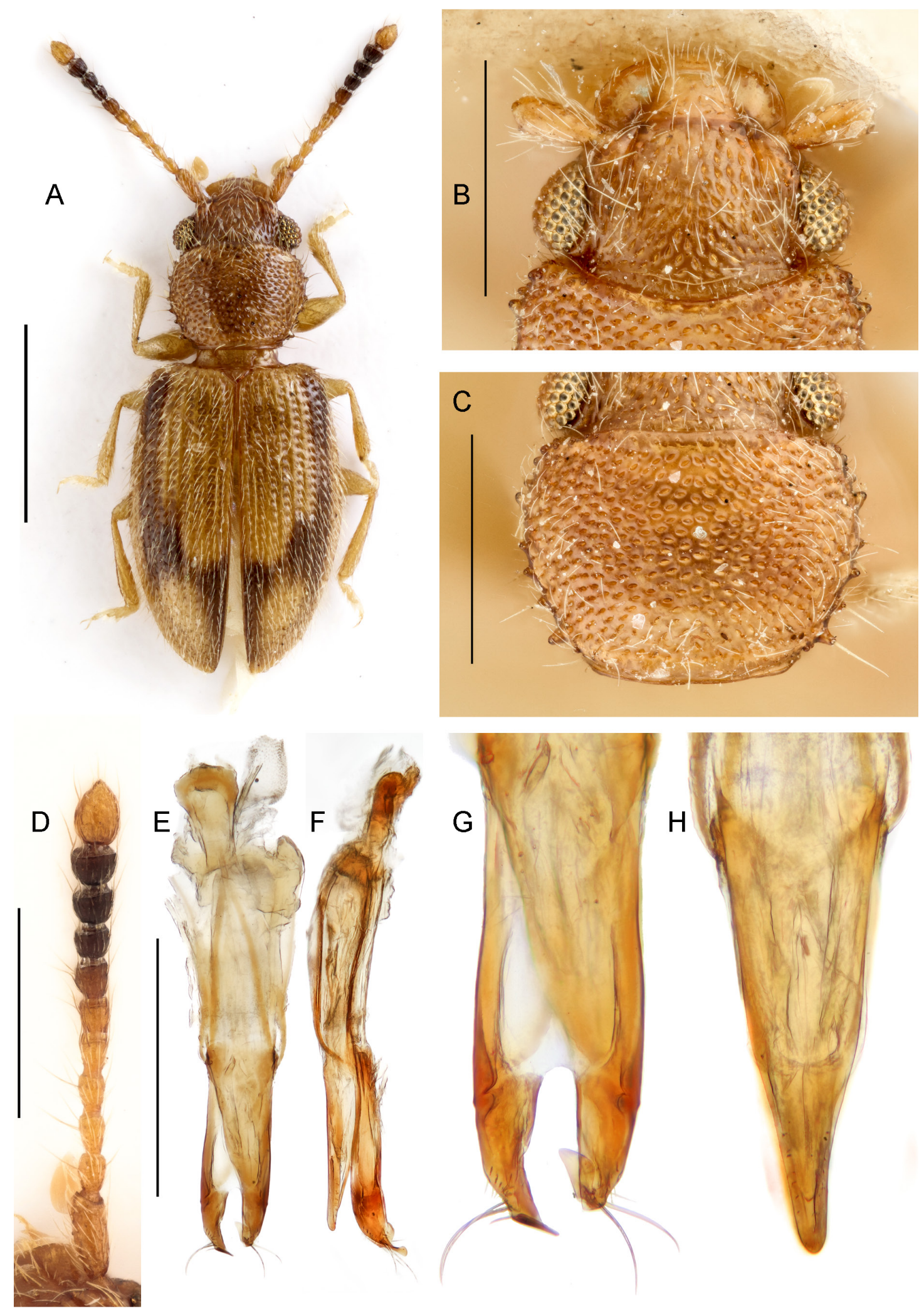

Fig. 5. Psammoecus vittifer Blackburn, 1903, §ิð. A, D. Specimen from Queensland (HNHM). B-C, E-H. Holotype (NHMUK). A. Habitus. B. Head. C. Pronotum. D. Right antenna. E. Aedeagus, ventral view. F. Aedeagus, lateral view. G. Parameres, ventral view. H. Median lobe, dorsal view. Scale bars: A $=1.0 \mathrm{~mm} ; \mathrm{B}-\mathrm{F}=0.5 \mathrm{~mm} ; \mathrm{G}-\mathrm{H}=0.2 \mathrm{~mm}$. 
rainforest, at light; ANIC • 1 spec.; City of Townsville, Townsville; 16-22 Mar. 1965; Balogh leg.;

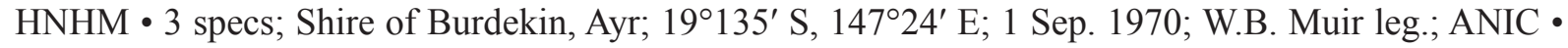

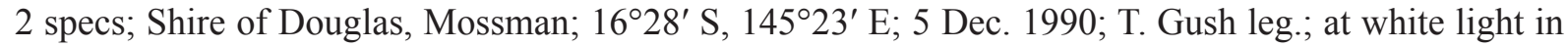
laundromat; ANIC • 1 spec.; Shire of Douglas, Wonga, Pinnacle Village Holiday Park; 0 m a.s.l.; 13 Jan. 1999; Podlussány leg.; HNHM • 1 spec.; Shire of Mareeba/Douglas Shire, Mount Lewis; 1010 m a.s.l.; 20 Jun. 1971; Taylor Feehan leg.; ANIC • 1 spec.; Shire of Mareeba, Kuranda, Barron Falls; 1649' S, $145^{\circ} 38^{\prime}$ E; 24 Nov. 1964; J.G. Brooks leg.; at light; ANIC.

THAILAND • 1 spec.; Isan, Khon-Kaen; 16² $26^{\prime}$ N, 102 ${ }^{\circ} 50^{\prime}$ E; 23 Feb. 1980; Saowakontha leg.; at light; $\mathrm{HNHM} \bullet 1 \mathrm{spm}$; same data as for preceding; MKF.

PAPUA NEW GUINEA • 1 spec.; Central Province, Ighibirei, Kemp Welch River; Jul.-Aug. 1890; Loria leg.; MSNG.

\section{Distribution}

This species was found in Australia (Queensland), Indonesia, Papua New Guinea and Thailand.

\section{Remarks}

Psammoecus vittifer is easily identified by its general habitus and the very distinct structure of the male genitalia. One specimen from Papua New Guinea in the MSNG collection is labelled as "Psammoecus ocularis ty. Grouv." in Grouvelle's hand. This name was never published, hence P. ocularis is considered a manuscript name.

\section{Psammoecus australis sp. nov. urn:Isid:zoobank.org:act:B8F383AA-30E8-4CE3-B033-600DEFFEF03E}

Fig. 6

\section{Diagnosis}

The following combination of character states distinguishes this species: body length 1.96-2.45 mm; eyes very large, evenly rounded; temples extremely short, strongly narrowed immediately behind eyes; frontal grooves diverging posteriorly, reaching middle of eyes; punctation on vertex very sparse; pronotal punctation sparse; pronotal teeth long; lateral pronotal margin simple; lateral elytral interstices with very long setae; median lobe wide, apex blunt, rounded; parameres short, roundish.

\section{Etymology}

The specific epithet is derived from the continent name Australia.

\section{Material examined}

\section{Holotype}

AUSTRALIA - 3 ; Queensland, Cook Shire, $30 \mathrm{~km} \mathrm{~S} \mathrm{of} \mathrm{Cooktown,} \mathrm{Mt} \mathrm{Finnigan} \mathrm{S1.;} 400$ m a.s.1.; 3 Jul. 1982; S. and J. Peck leg.; rainforest, moist litter; ANIC.

\section{Paratypes}

AUSTRALIA - Queensland - Cairns Region • 1 spec.; Bellenden, Ker Range, Cable Tower 3; 1054 m

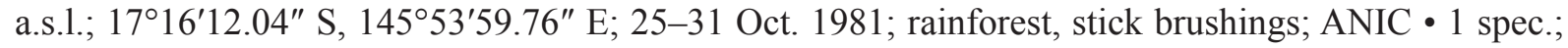
same locality; 17 Oct.-9 Nov. 1981; pyrethrum knockdown; ANIC • 1 spec.; Bellenden, Ker Range, Cableway Base Station; 100 a.s.1.; $17^{\circ} 16^{\prime}$ S, 145 ${ }^{\circ} 54^{\prime}$ E; 25-31 Oct. 1981; rainforest, sieved litter; ANIC - 10 specs; same locality; 17-24 Oct. 1981; rainforest, sieved litter; ANIC $\bullet 3$ specs; same data as for preceding; MKF • 2 specs; Bellenden, Ker Range, Cableway Base Station; 100 m a.s.l.; 17²16'12.04" S, 
14553'59.76" E; 25-31 Oct. 1981; rainforest, sieved litter; ANIC • 2 specs; Mulgrave River Road, 7 km

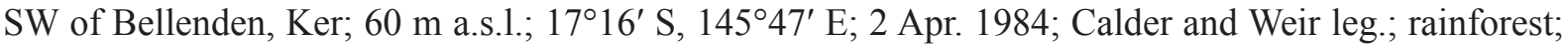
ANIC. - Cassowary Coast Region - 1 spec.; Mission Beach; 10 m a.s.1.; 17050' S, $146^{\circ} 06^{\prime}$ E; 29 Jan.-4 Mar. 1996; Cermak leg.; Malaise trap; ANIC - 2 specs; Mission Beach; 40 m a.s.1.; 17 $52^{\prime}$ S, 14604' E; 29 Jan.-4 Mar. 1996; Cermak leg.; flight intercept trap; ANIC • 1 spec.; same locality; 4 Mar.-1 Apr. 1996; Cermak leg.; flight intercept trap; ANIC. Shire of Douglas 22 specs; 2 km SSW of Cape Tribulation; $10 \mathrm{~m}$ a.s.l.; $16^{\circ} 05^{\prime} \mathrm{S}, 145^{\circ} 28^{\prime} \mathrm{E}$; 28 Feb.-27 Mar. 1996; Zborowski leg.; flight intercept trap; ANIC 11 spec.; 1 km WNW of Cape Tribulation; $10 \mathrm{~m}$ a.s.1.; $16^{\circ} 04^{\prime} \mathrm{S}, 145^{\circ} 28^{\prime} \mathrm{E}$; 5 Dec. 1995-4 Jan. 1996; Umback leg.; flight intercept trap; ANIC • 1 spec.; 2 km WSW of Cape Tribulation; $30 \mathrm{~m}$ a.s.l.; $16^{\circ} 05^{\prime} \mathrm{S}, 145^{\circ} 27^{\prime} \mathrm{E}$; 4 Jan.-1 Feb. 1996; Umback leg.; pitfall trap; ANIC 1 spec.; same locality; 1-28 Feb. 1996; Umback leg.; pitfall trap; ANIC - 1 spec.; same locality; 28 Feb.-27 Mar. 1996; Zborowski leg.; flight intercept trap; ANIC • 4 specs; Cape Tribulation Area; $16^{\circ} 03^{\prime} \mathrm{S}, 145^{\circ} 28^{\prime} \mathrm{E}$; 1-11 May 1992; Lawrence leg.; leaf and log litter; ANIC • 2 specs; 45 km N of Port Douglas, Cape Tribulation; $16^{\circ} 03^{\prime} \mathrm{S}, 145^{\circ} 28^{\prime} \mathrm{E}$; 21-28 Mar. 1984; Calder and Weir leg.; lowland rainforest; ANIC • 2 specs; 40 km N of Daintree, Cape Tribulation; 10 m a.s.l.; 12 Jul. 1982; S. and J. Peck leg.; rainforest, leaf and $\log$ litter; ANIC $1 \mathrm{spm}$; same data as for preceding; MKF $\bullet 1 \mathrm{spec}$.; Shire of Cook, Moses

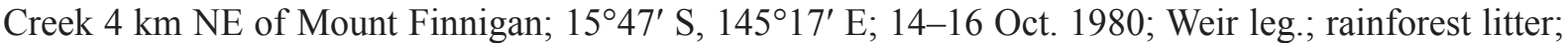
ANIC - 1 spec.; Shire of Cook, 30 km S of Cooktown, Mt Finnigan; 400 m a.s.l.; 1 Jul. 1982; S. and J. Peck leg.; rainforest, litter and fungi; ANIC • 1 spec.; Kuranda, Black Mountain Road; 390 m a.s.l.; $17^{\circ} 26^{\prime}$ S, $145^{\circ} 35^{\prime}$ E; 22 Jun. 1971; Feehan leg.; rainforest; ANIC • 1 spec.; Kuranda, Black Mountain Road; 350 m a.s.1.; 27 Jun. 1971; Feehan leg.; ANIC • 1 spec.; Shire of Cook, 3 km NE of Mount Webb; $15^{\circ} 03^{\prime} \mathrm{S}, 145^{\circ} 09^{\prime}$ E; 1-3 Oct. 1980; Weir leg.; rainforest litter; ANIC 3 specs; Shire of Cook, Cape York Peninsula, Mount Webb National Park; $15^{\circ} 04^{\prime}$ S, $145^{\circ} 07^{\prime}$ E; 28-30 Sep. 1980; Weir leg.; rainforest litter; ANIC - 1 spec.; Shire of Douglas, Mossman Gorge National Park, 6 km SW of Mossman; 50 m a.s.l.; 11 Jul. 1982; S. and J. Peck leg.; rainforest leaf litter; ANIC • 3 specs; Shire of Douglas, Daintree, Pilgrims Sands; 10 m a.s.1.; 20-27 Jan. 1991; Hammond leg.; lowland tropical forest, flight interception trap; NHMUK • $1 \mathrm{spm}$; same data as for preceding; MKF 2 specs; Shire of Lockhart River, 9 km ENE of Mount Tozer; $12^{\circ} 43^{\prime}$ S, $143^{\circ} 17^{\prime}$ E; 5-10 Jul. 1986; Weir leg.; rainforest litter; ANIC.

\section{Description of holotype}

Body oval, stout, $2.13 \mathrm{~mm}$ long; testaceous (Fig. 6A); elytra with dark maculae near middle and near apex; antennomeres 8-9 slightly and 7 very slightly darkened, antennomeres $10-11$ whitish (Fig. 6D). Head (Fig. 6B) $0.36 \mathrm{~mm}$ long, $0.55 \mathrm{~mm}$ wide; frontal grooves distinct, deep, curved outwards toward posterior end, reaching middle of eyes; vertex with sparse punctation, punctures small; setae of very uneven lengths, directed straight forward, seta near end of each frontal groove almost as long as eyes, shortest setae only as long as two eye facet diameters; microsculpture absent; eyes large, strongly protruding, unevenly rounded, $0.16 \mathrm{~mm}$ long, eye distance $0.32 \mathrm{~mm}$; temples absent, head narrowed immediately behind eyes, temple angle $83^{\circ}$; antennae (Fig. 6D) long, slender, length $1.29 \mathrm{~mm}$, antennomere proportions $3.0: 1.1: 1.3: 1.4: 1.5: 1.4: 1.4: 1.2: 1.1: 1.0: 2.7$. Pronotum (Fig. 6C) $0.63 \mathrm{~mm}$ wide, $0.43 \mathrm{~mm}$ long, widest at anterior third; at anterior denticles $1.32 \times$ as wide as at posterior denticles; very shallowly impressed near posterior margin; lateral margin slightly ridged between lateral teeth III and V, with distinct group of three anterior denticles and additional small denticles along anterior pronotal margin, lateral margin with distinct teeth; I small, only slightly larger than anterior denticles, II to IV of increasing length, V as large as III; posterior denticle distinct, slightly smaller than anterior denticles; punctation on pronotal disc slightly denser than on vertex, punctures somewhat smaller than eye facet diameter; setae on pronotal disc of very uneven lengths, directed anteromedially, longest setae about $3 / 5$ as long as eyes, shortest $1.5 \times$ as long as eye facet diameter.

Elytra (Fig. 6A) oval, $1.29 \mathrm{~mm}$ long, $0.94 \mathrm{~mm}$ wide, striae twice as wide as interstices, pubescence semierect, interstrial setae about twice as long as strial setae, even longer near elytral margins; interstice 

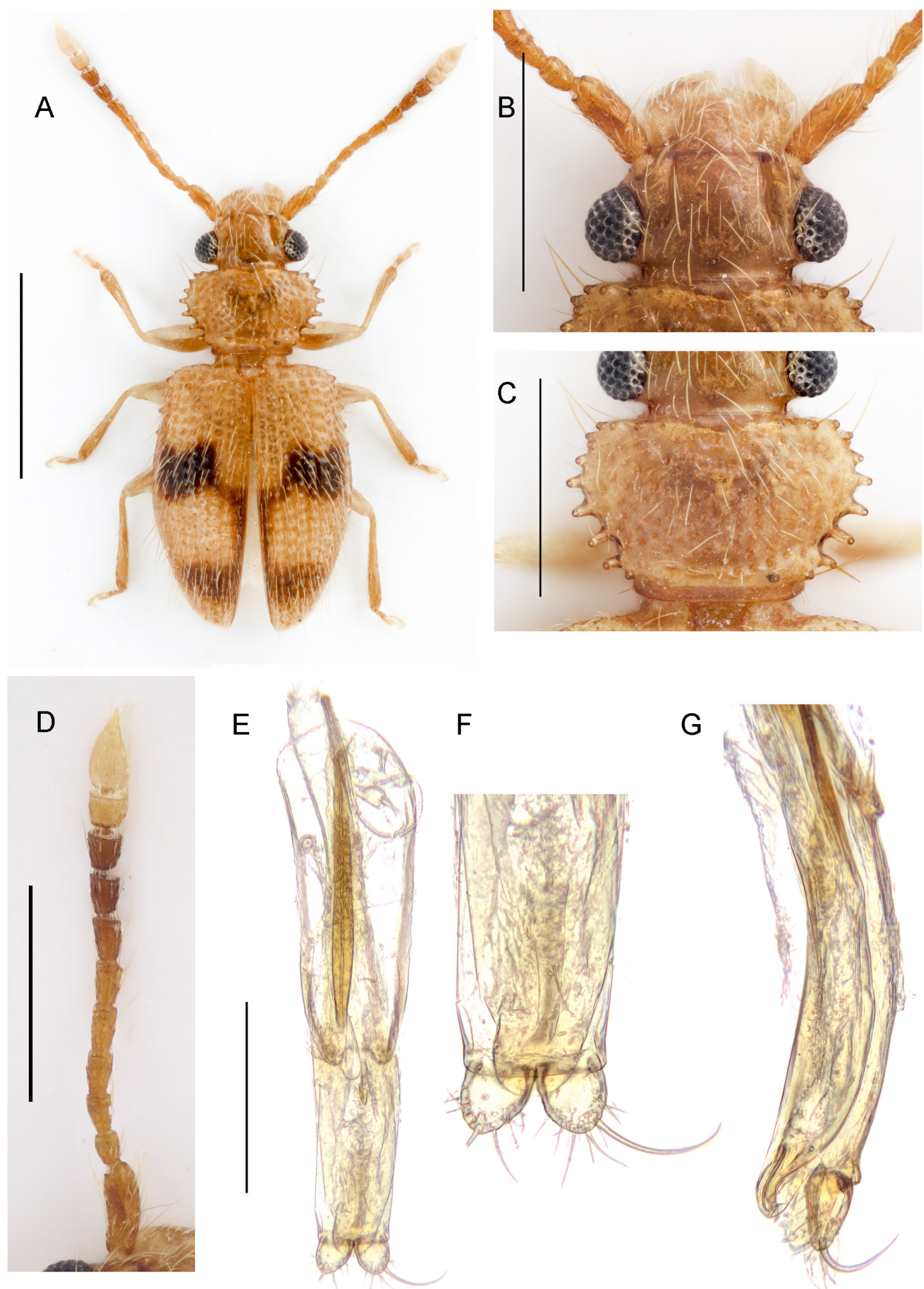

Fig. 6. Psammoecus australis sp. nov, $\widehat{\jmath}$. A-C. Holotype (ANIC). A. Habitus. B. Head. C. Pronotum. D. Left antenna. E-G. Paratype from Queensland (ANIC). E. Aedeagus, ventral view. F. Parameres, ventral view. G. Parameres and median lobe, lateral view. Scale bars: $A=1.0 \mathrm{~mm}$; $-\mathrm{D}=0.5 \mathrm{~mm}$; E = $0.2 \mathrm{~mm} ; \mathrm{F}-\mathrm{G}=0.1 \mathrm{~mm}$. 
between $9^{\text {th }}$ and $11^{\text {th }}$ stria with distinct denticles near humerus; microsculpture absent. Male genitalia (Fig. 6E-G) with wide median lobe, apex evenly rounded, in the shape of a half-circle, parameres short, stout, almost circular in ventral view, each with one very long and several shorter setae.

\section{Distribution}

Australia (Queensland).

\section{Biology}

The species was found in flight intercept traps, in berlese funnels and in plant detritus.

\section{Variation}

Paratypes range in length: $1.96-2.45 \mathrm{~mm}$. The elytral maculae differ considerably in size. In some specimens, only the $11^{\text {th }}$ antennomere is whitish.

\section{Remarks}

Psammoecus australis sp. nov. is closely related to Psammoecus delicatus Grouvelle, 1908 and Psammoecus complexus Pal, 1985. It can be distinguished from P. complexus by the distinctly different colouration, and from both species by the different shape of lateral pronotal teeth and male genitalia.

Psammoecus venustus sp. nov. urn:1sid:zoobank.org:act:2453FD2F-D632-4915-9692-ACFF3FE346B9

Fig. 7

\section{Diagnosis}

The following combination of character states distinguishes this species: body length $2.32-3.14 \mathrm{~mm}$; eyes moderately sized, evenly rounded; temples well developed, evenly rounded; frontal grooves parallel posteriorly, almost reaching middle of eyes; punctation on vertex sparse; pronotal punctation moderate; pronotal teeth short; lateral pronotal margin ridged; lateral elytral interstices with very long setae; median lobe slender, lancet-shaped; parameres slender, parallel-sided, curved.

\section{Etymology}

The specific epithet was chosen in reference to the beautiful habitus of this species.

\section{Material examined}

Holotype

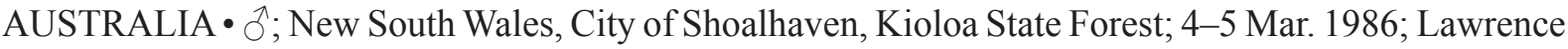
leg.; rainforest, leaf and log litter; ANIC.

\section{Paratypes}

AUSTRALIA - New South Wales 2 specs; Canberra, $3.5 \mathrm{~km}$ E of Piccadilly Circus ACT, Blundells Creek Road; 850 m a.s.l.; 27 Jan. 1982; Lawrence leg.; leaf and log litter; ANIC • 1 spec.; Sydney; ANIC - 10 specs; Armidale Dumaresq Council, Kookaburra, Carrai National Park; 943 m a.s.1.; 31 $01^{\prime} 434^{\prime \prime}$ S, $152^{\circ} 20^{\prime} 288^{\prime \prime}$ E; 27-28 Oct. 2000; Podlussány leg.; HNHM • 1 spec.; Bellingen Shire, 6 km NNE of Raleigh, Pine Creek State Forest; $30^{\circ} 24^{\prime}$ S, $153^{\circ} 01^{\prime}$ E; 16 Nov. 1976; Common and Edwards leg.; ANIC - 1 spec.; Blue Mountains, Mount Wilson; 14 Mar. 1978; HNHM • 1 spec.; Bombala Council, 4 km NE of Wog Wog, $17 \mathrm{~km} \mathrm{SE}$ of Bombala; 31 ${ }^{\circ} 04^{\prime} 30^{\prime \prime} \mathrm{S}, 149^{\circ} 28^{\prime} 00^{\prime \prime}$ E; Oct. 1995; Milkovits leg.; pitfall trap;

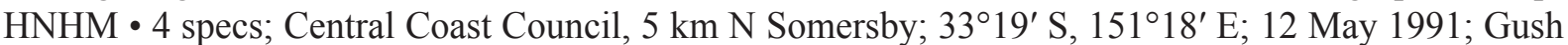
leg.; leaf litter; ANIC • 3 specs; Central Coast Council, Strickland State Forest; $33^{\circ} 23^{\prime}$ S, $151^{\circ} 19^{\prime}$ E; 

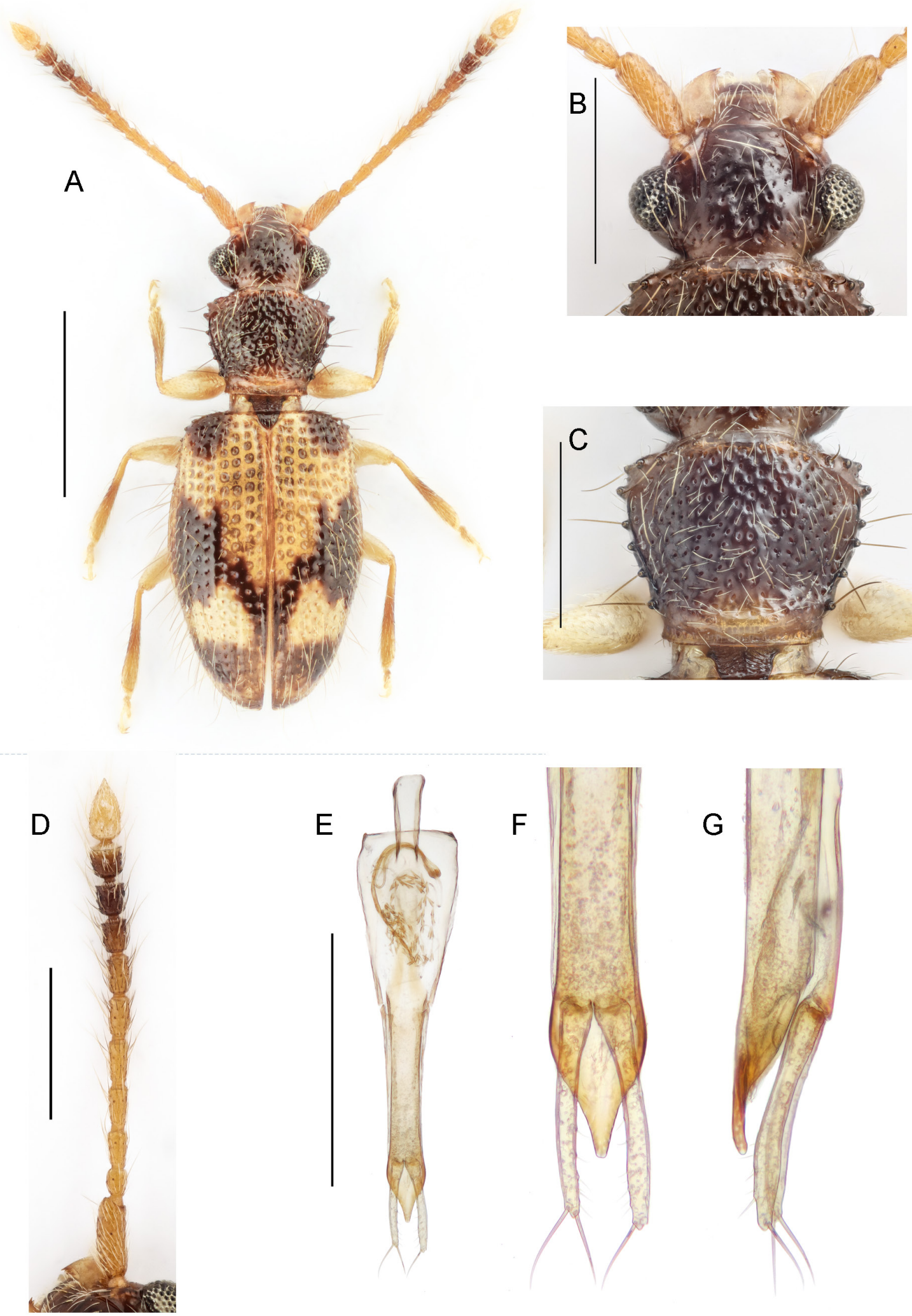

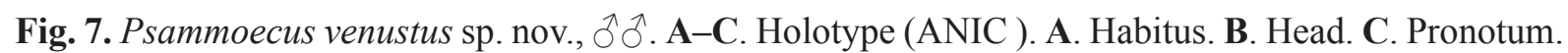
D. Right antenna. E-G. Paratype spec. from Queensland (ANIC). E. Aedeagus, ventral view. F. Parameres, ventral view. G. Parameres and median lobe, lateral view. Scale bars: A $=1.0 \mathrm{~mm}$; B-E = $0.5 \mathrm{~mm} ; \mathrm{F}-\mathrm{G}=0.2 \mathrm{~mm}$. 
26 May 1991; Gush leg.; leaf litter; ANIC • 1 spec.; City of Blue Mountains, Wentworth Falls; 16 Jan. 1909; Bryant leg.; NHMUK • 1 spec.; City of Coffs Harbour, Bruxner Park, Park Creek Picknick site; $30^{\circ} 14^{\prime} \mathrm{S}, 153^{\circ} 06^{\prime} \mathrm{E}$; 6 Apr. 1993; Reid leg.; rainforest, beaten from vegetation; ANIC 11 spec.; City of

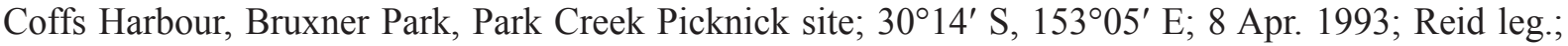
rainforest, beaten from vegetation; ANIC -1 spec.; City of Maitland, Brown Mountain, Rutherford Creek; 15 Jan. 1969; Curtis leg.; ANIC 11 spec.; City of Shoalhaven, Barrengarry Mountain; 460 m a.s.1.; 28 Dec. 1966; rainforest, leaf mould; ANIC 1 spec.; City of Shoalhaven, Barrengarry Mountain, 24 km SE of Mossvale; 600 m a.s.l.; 29 Aug. 1982; S and J Peck leg.; rainforest, log and leaf litter; ANIC - 1 spec.; City of Shoalhaven, Bawley Point; 35³0' S, 150 24' E; 14 Nov. 1993; Rentz and MacCarron leg.; at light; MKF • 1 spec.; Dungog Shire, Upper Allyn Road; 22 Apr. 1970; Colless leg.; ANIC • 1 spec.; Dungog Shire, Upper Williams River; Oct. 1926; Lea and Wilson leg.; ANIC - 3 specs;

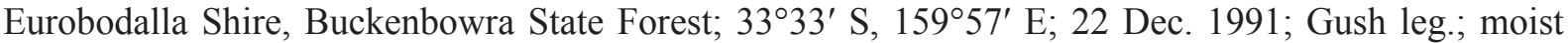
debris in creek; ANIC • 1 spec.; Illawarra; 13 Sep. 1908; Bryant leg.; NHMUK • 1 spec.; same locality; 27 Sep. 1908; Bryant leg.; NHMUK• 2 specs; same locality; 2 Oct. 1908; Bryant leg.; NHMUK, 1 spm; same data as for preceding; MKF • 4 specs; same locality; Oct. 1908; Bryant leg.; NHMUK • 1 spec.; same locality; Nov. 1908; Bryant leg.; NHMUK • 3 specs; Kyogle Council, Acacia Plateau; Armstrong leg.; ANIC • 4 specs; Mid North Coast, Kerewong; 21 Dec. 1978; HNHM • 2 specs; Mid-Coast Council, $3 \mathrm{~km}$ NE of Wootton; $32^{\circ} 16^{\prime} \mathrm{S}, 152^{\circ} 19^{\prime} \mathrm{E}$; 22 Sep. 1991; Gush leg.; leaf litter at edge of river; ANIC • 1 spec.; Mid-Coast Council, Landsdowne State Forest; 31 ${ }^{\circ} 44^{\prime}$ S, 152 ${ }^{\circ} 31^{\prime}$ E; 22 May 1992; Gush leg.; creek debris; ANIC • 1 spec.; Mid-Coast Council, Taree, 3 km N of Landsdowne; 19-26 Sep. 1992; Williams leg.; riparian rainforest, Malaise trap; ANIC $\bullet 1$ spec.; Mid-Coast Council, Lorien Wildlife Refuge, Landsdowne, Taree; Dec. 1986; H. and A. Howden leg.; flight intercept trap; ANIC • 1 spec.; Mid-Coast Council, Wang Wauk State Forest; 32²0' S, 152 ${ }^{\circ} 15^{\prime}$ E; 22 Sep. 1991; Gush leg.; leaf litter in creek bed; ANIC 1 spec.; Municipality of Kiama, 13 km W of Kiama, Jamberoo Pass; 27 Feb. 1969; Britton, Upton and Misko leg.; ANIC - 1 spec.; Queanbeyan-Palerang Regional Council, Clyde Mountain; 750 m a.s.1.; 4 Dec. 1967; Taylor and Brooks leg.; wet sclerophyll; ANIC • 1 spec.; Queanbeyan-Palerang Regional Council, 6 km SSE of Monga; Oct. 1989; Reid leg.; beating RF bushes; ANIC 1 spec.; Queanbeyan-Palerang, 4 km N of Mongarlowe; 25 May 1991; Reid leg.; on shrubs by river; ANIC • 1 spec.; Southern Tablelands Region, Clyde Mountain; 16 Mar. 1977; Moore leg.; ANIC - 1 spec.; Southern Tablelands Region, Clyde Mountain, Cabbage Tree Creek; 27 Sep. 1979; Naumann and Cardale leg.; ANIC • 1 spec.; Tenterfield Shire Council, Unumgar S. F., Grevillia, Coxs Road; $580 \mathrm{~m}$ a.s.l.; $28^{\circ} 27^{\prime} \mathrm{S}, 152^{\circ} 45^{\prime} \mathrm{E}$; 2-11 Jan. 1987; Newton and Thayer leg.; subtropical rainforest, flight intercept trap; ANIC 1 spec.; Tenterfield Shire Council, Unumgar S. F., near Woodenbong, Pole Bridge Road; 430 m a.s.1.; $28^{\circ} 24^{\prime} \mathrm{S}, 152^{\circ} 40^{\prime} \mathrm{E}$; 2-11 Jan. 1987; Newton and Thayer leg.; dry rainforest (Araucaria, Eucalyptus), flight intercept trap; ANIC. - Queensland - 1 spec.; Atherton Tableland,

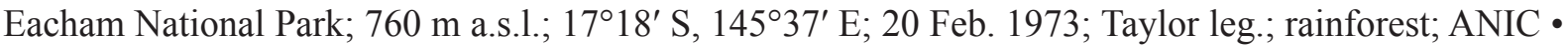
1 spec.; Cairns Region, 22 km from Cairns, Whitfield Road; 670 m a.s.1.; 21 Oct. 1971; Brooks leg.; ANIC 11 spec.; Cassowary Coast Region, Cardstone; 10 Dec. 1966; Brooks leg.; ANIC 2 specs; Cassowary Coast Region, Tully Falls; 15 Dec. 1976; Walford-Huggins leg.; NHMUK • 1 spec.; Charters Towers Region, 19 km W of Paluma, Ewan Road; 6 Jan. 1966; J.G. and J.A.G. Brooks leg.; ANIC • 1 spec.; Charters Towers Region, Mount Spec; 880 m a.s.l.; $18^{\circ} 55$ S, 14609’ E; 8 May-5 Jun. 1995; Cermak leg.; flight intercept trap; ANIC • 1 spec.; same locality; 5 Jun.-3 Jul. 1995; Cermak leg.; flight intercept trap; ANIC • 1 spec.; same locality; 4 Sep.-1 Oct. 1995; Cermak leg.; flight intercept trap; ANIC • 1 spec.; same locality; 1 Oct.-4 Nov. 1995; Cermak leg.; flight intercept trap; MKF • 3 specs; same locality; 4 Nov.-1 Dec. 1995; Cermak leg.; flight intercept trap; ANIC 1 spec.; Charters Towers Region, Mount Spec; 880 m a.s.l.; $18^{\circ} 55$ S, 146 $16^{\circ}$ E; 9 Mar.-6 Apr. 1995; Cermak leg.; flight intercept trap; ANIC - 3 specs; same locality; 4 Nov.-1 Dec. 1995; Cermak leg.; flight intercept trap; ANIC • 1 spec.; City of Townsville, Townsville; Jul. 1921; Carter leg.; ANIC 11 spec.; City of Townsville, 6 km WNW of Paluma, Birthday Creek; $18^{\circ} 59^{\prime}$ S, $146^{\circ} 10^{\prime}$ E; 25 Sep. 1980; Weir leg.; leaf and log litter; ANIC • 1 spec.; Hichinbrook Shire Council, Ingham; 22-28 Mar. 1965; Balogh leg.; HNHM • 1 spec.; 
Mackay Region, Eungella; Naumann and Cardale leg.; ANIC • 1 spec.; Moreton Bay Region, Miala National Park; $27^{\circ} 20^{\prime}$ S, $152^{\circ} 46^{\prime}$ E; 13 Mar. 1973; rainforest; ANIC 11 spec.; Moreton Bay Region, Mount Glorious; 630 m a.s.1.; 14 Nov. 1986-30 Jan. 1987; Hiller leg.; rainforest, flight intercept trough trap; ANIC • 1 spec.; Scenic Rim Region, Joalah National Park, Tamborine Mountain; 23 Jul. 1976; Lawrence leg.; litter and flood debris; ANIC • 2 specs; Shire of Mareeba, Kuranda; May 1970; Brooks leg.; ANIC • 1 spec.; Shire of Mareeba, Massey Creek; 1000 m a.s.l.; 17³7' S, 135³4' E; 2 Jun.-4 Jul. 1995; Zborowski leg.; flight intercept trap; ANIC • 1 spec.; same locality; 4 Jul.-2 Aug. 1995; Zborowski leg.; flight intercept trap; ANIC • 1 spec.; same locality; 2-30 Nov. 1995; Umback leg.; flight intercept trap; ANIC • 3 specs; Shire of Mareeba, Kuranda, Lamb Range; 23 May 1970; Brooks leg.; ANIC • 1 spec.; Shire of Mareeba, Windsor Tableland, Mt Carbine; 9 Sep.-4 Nov. 1976; Storey leg.; rainforest, pitfall trap; NHMUK • 1 spec.; Tablelands Region, Evelyn; May 1954; Brooks leg.; ANIC • 1 spec.; Tablelands Region, Hugh Nelson Range; $17^{\circ} 27^{\prime}$ S, 145 $29^{\prime}$ E; 30 Nov. 1993; Umback leg.; flight intercept trap; ANIC $11 \mathrm{spec}$.; Tablelands Region, Wongabel S.F., 6 km S of Atherton; 1 Dec. 19839 Jan. 1984; Storey and Brown leg.; flight intercept trap; NHMUK 11 spec.; Tablelands Region, Barrine National Park; 760 m a.s.l.; 21 Mar. 1975; Taylor leg.; ANIC • 2 specs; Tablelands Region, 8 km N of Gillies Highway, Boar Pocket Road; 13 Dec. 1969; Brooks leg.; leaf litter; ANIC • 1 spec.; Tablelands

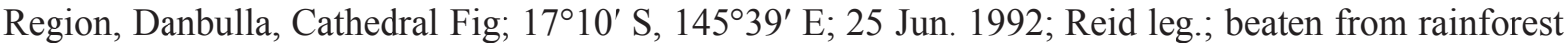
trees and vines; ANIC • 1 spec.; Tablelands Region, Sm. Tinaroo Falls, Danbulla Road; 26 Apr. 1967; Colless leg.; at light; ANIC • 1 spec.; Tablelands Region, Mount Baldy near Atherton, Forest Reserve No. 194; 1200 m a.s.1.; 5 Dec. 1968; Britton and Misko leg.; rainforest; MKF • 1 spec.; Tablelands Region, Lake Barrine; 720 m a.s.1; 25 Feb. 1970; Brooks leg.; ANIC • 1 spec.; Tablelands Region, Wongabel State Forest, via Atherton; 19-20 May 1970; Naumann and Cardale leg.; ANIC • 1 spec.; Tablelands Region, Alberton, Wongabel State Forest; 19-20 May 1980; Naumann and Cardale leg.; MKF • 7 specs; Townsville City, Townsville; 16-22 Mar. 1965; Balogh leg.; HNHM. - Victoria • 1 spec.; Shire of East Gippsland, Croajingolong National Park, South-West Arm Road, 11 km NW of Mallacoota; 20 m a.s.1.; 37³1' S, 149 41' E; 10-23 Feb. 1993; Newton and Thayer leg.; Banksia woodland, window trap; ANIC • 1 spec.; Shire of East Gippsland, Lind National Park, Euchre Valley, Olive Branch Creek; $140 \mathrm{~m}$ a.s.1.; 37³4' S, 148 57' E; 10-23 Feb. 1993; Newton and Thayer leg.; riparian forest, window trap; ANIC 11 spec.; Shire of East Gippsland, $12 \mathrm{~km} \mathrm{~N}$ of Orbost, Bonang Highway, Young's Creek; 9 Nov. 1976; Colless and Liepa leg.; ANIC • 2 specs; South Gippsland Shire, Wilson's Promontory National Park, Lilly Pilly Tr. VIC; 14 May 1978; S. and J. Peck leg.; ANIC.

\section{Description of holotype}

Body (Fig. 7A) elongate, length $2.71 \mathrm{~mm}$; yellowish brown; head piceous, pronotum piceous, narrow region at anterior margin lighter brown, wider, distinctly yellowish brown region across posterior margin, elytra with piceous maculae: humeral area, irregular maculae near middle, and apex dark, scutellar shield piceous, antennomeres 8-10 darkened, antennomere 11 lighter; middle of tibiae slightly darkened. Head (Fig. 7B) $0.4 \mathrm{~mm}$ long, $0.64 \mathrm{~mm}$ wide; frontal grooves distinct, deep, converging anteriorly, parallel posteriorly, almost reaching middle of eyes; vertex with sparse punctation; punctures distinctly smaller than eye facet diameter, somewhat longitudinally stretched; setae of variable length, mostly about $1 / 3$ as long as eyes, directed anteromedially; microsculpture absent; eyes of moderate size, evenly rounded, $0.18 \mathrm{~mm}$

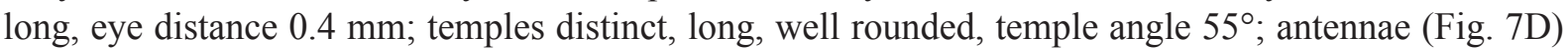
long, slender, $1.69 \mathrm{~mm}$ long, antennomere proportions $2.9: 1.0: 1.4: 1.5: 1.6: 1.4: 1.4: 1.2: 1.1: 1.1: 2.3$. Pronotum (Fig. 7C) $1.1 \times$ as wide as long, widest at anterior quarter; at anterior denticles $1.3 \times$ as wide as at posterior denticles; lateral margin almost straight along posterior $3 / 4$, with ridge between anterior and posterior denticles; anterior end of ridge curved medially, $0.63 \mathrm{~mm}$ wide, $0.57 \mathrm{~mm}$ long, with group of three anterior denticles, lateral margin with distinct, short teeth, I slightly larger than II-IV, posterior denticle very distinct, almost as large as I, punctation on pronotal disc moderate, punctures slightly larger and denser, as on vertex; slightly stretched longitudinally; setae as on vertex, directed anteromedially; microsculpture absent. Elytra (Fig. 7A) elongate oval, widest at middle, $1.60 \mathrm{~mm}$ long, 
$1.04 \mathrm{~mm}$ wide, striae about as wide as interstices, pubescence strial setae $1 / 3$ to $1 / 2$ as long as interstrial setae; lateral insterstices with very long, erect setae; microsculpture absent. Male genitalia (Fig. 7E-G) with slender, lancet shaped median lobe, internal sac with numerous small denticles and long, curved ductus; parameres long, slender, parallel-sided, slightly curved medially, with two large apical setae and numerous setae along the inner margins.

\section{Variation}

Paratypes range in length from $2.32 \mathrm{~mm}$ to $3.14 \mathrm{~mm}$. The elytral colouration varies considerably in the size of the dark maculae, but with the general pattern of colouration present in all specimens. The colour of head and pronotum ranges from entirely testaceous to piceous. The $10^{\text {th }}$ antennomere is often whitish, like the $11^{\text {th }}$.

\section{Distribution}

Australia (Canberra, New South Wales, Queensland, Victoria).

\section{Biology}

The species was found on plants, in plant detritus, in flight intercept and pitfall traps and and only once at light.

\section{Remarks}

This species somewhat resembles Psammoecus felix (Waterhouse, 1876), described from Sri Lanka. Psammoecus venustus differs distinctly in colouration, in the shape of the male genitalia, and in the structure of the frontal grooves.

\section{Discussion}

The present paper is the first to provide a revision of all species of Psammoecus known from Australia. It records seven species, two of them new to science and one new to Australia. Surprisingly to the author, no Australian specimen of Psammoecus trimaculatus was among the numerous specimens that were studied, even though it is a very widely distributed species, most likely by human trade (see Mola \& Yoshida 2019 for an overview on the current knowledge about its distribution).

Several species of the genus show extraordinarily large areas of distribution (Mola \& Yoshida 2019; Yoshida 2018). The three exclusively Australian species (P. australis sp. nov., P. venustus sp. nov., $P$. incertior) do not represent a distinct group within the genus, and a look at all seven species reveals a quite typical representation of Psammoecus diversity. It can not be ruled out that the current Australian fauna of Psammoecus is a mixture of mostly imported species. Human trade is likely an important factor for the spread of many species of Psammoecus (see also Lu \& Han 2006; Ouellette 2018); their association with plant detritus and high flight activity corroborates this hypothesis.

However, the generally poor knowledge about the genus and lack of faunistic data do not allow for more than speculation. Future studies on the Psammoecus of Australia and adjacent areas might shed light on this question.

\section{Acknowledgements}

For their very generous loan of material I am indebted to the following colleagues: Adam Slipinski (ANIC); Maxwell Barclay, Roger Booth and Michael Geiser (NHMUK); Ottó Merkl (HNHM); Azadeh Taghavian (MNHN); Roberto Poggi (MSNG); Hans Huijbregts and Pasquale Ciliberti (RMNH). 


\section{References}

Arrow G. J. 1927. Clavicornia, Cucujidae. In: Insects of Samoa and other Samoan terrestrial Arthropoda. Part IV Fasc. 1: 42-47. British Museum (Natural History), London.

Available from https://www.biodiversitylibrary.org/page/43252305 [accessed 19 Oct. 2020].

Blackburn T. 1903. Further notes on Australian Coleoptera, with descriptions of new genera and species. Transactions of the Royal Entomological Society of South Australia 27: 91-182.

Grouvelle A. 1908. Coléoptères de la région indienne. Annales de la Société entomologique de France 77: 315-495.

Grouvelle A. 1912. Psammoecus nouveaux du Musée de Leide. Notes from the Leyden Museum 34: 81-94.

Grouvelle A. 1919. Descriptions d'espèces nouvelles du genre Psammoecus. Mémoires entomologiques. Études sur les Coléoptères. Deuxième Fascicule. Société entomologique de France, Paris.

Hetschko A. 1930. Fam. Cucujidae. In: Junk W. \& Schenkling S. (eds) Coleopterorum Catalogus 109: 3-107. W. Junk, Berlin.

Karner M. 2012. A revision of African Psammoecus from the collection of the Musée royal de l'Afrique centrale. European Journal of Taxonomy 17: 1-31. https://doi.org/10.5852/ejt.2012.17

Karner M. 2014. Three new species and new records of African Psammoecus Latreille (Coleoptera, Silvanidae). European Journal of Taxonomy 89: 1-18. https://doi.org/10.5852/ejt.2014.89

Lu Y. \& Han Z. 2006. Five narrowly distributed species of Silvanidae from Yangzhou captured in wet blue leather and packages. Chinese Bulletin of Entomology 43: 398-400.

Mola L. \& Yoshida T. 2019. Psammoecus trimaculatus Motschulsky, 1858, new to the Italian fauna (Cucujoidea; Silvanidae). Fragmenta Entomologica 51 (1): 47-50. https://doi.org/10.4081/fe.2019.341

Ouellette G.D. 2018. Intercepted Silvanidae (Insecta: Coleoptera) from the International Falls, MN (U.S.A.) Port of Entry. Great Lakes Entomologist 51: 5-9.

Pal T.K. 1985. A revision of Indian Psammoecus Latreille (Coleoptera: Silvanidae). Records of the Zoological Survey of India 71: 1-54.

Waterhouse C.O. 1876. Descriptions of new species of Cucujidae and Cleridae. The Entomologist's Monthly Magazine 13: 118-129.

Yoshida T. \& Hirowatari T. 2014. A revision of Japanese species of the genus Psammoecus Latreille (Coleoptera, Silvanidae). ZooKeys 403: 15-45. https://doi.org/10.3897/zookeys.403.7145

Yoshida T., Karner M. \& Hirowatari T. 2018. A revision of Taiwanese species in the genus Psammoecus Latreille (Coleoptera, Silvanidae). Zoological Studies 57: 18. https://doi.org/10.6620/ZS.2018.57-18

Manuscript received: 30 March 2020

Manuscript accepted: 4 August 2020

Published on: 12 November 2020

Topic editor: Nesrine Akkari

Section editor: Max Barclay

Desk editor: Chloë Chester 
Printed versions of all papers are also deposited in the libraries of the institutes that are members of the EJT consortium: Muséum national d'histoire naturelle, Paris, France; Meise Botanic Garden, Belgium; Royal Museum for Central Africa, Tervuren, Belgium; Royal Belgian Institute of Natural Sciences, Brussels, Belgium; Natural History Museum of Denmark, Copenhagen, Denmark; Naturalis Biodiversity Center, Leiden, the Netherlands; Museo Nacional de Ciencias Naturales-CSIC, Madrid, Spain; Real Jardín Botánico de Madrid CSIC, Spain; Zoological Research Museum Alexander Koenig, Bonn, Germany; National Museum, Prague, Czech Republic. 